\title{
Magnetocardiography in the determination of fetal heart rate complexity
}

\author{
Van Leeuwen, P.1, Bettermann, H.², Schüßler, M.3, Lange, S.1 \\ Department of Biomagnetism, Research and Development Center for Microtherapy (EFMT), \\ Bochum, Germanyl, Institute for Mathematics, University of Witten/Herdecke, Witten, Germany², \\ Department of Obstetrics, Marienhospital, Witten, Germany ${ }^{3}$
}

\section{Introduction}

Heart rate variability (HRV) is an important marker for fetal well-being. Various methods have been used to analyse HRV, in particular parameters from the time and frequency domain [1]. Recently mathematical methods have been introduced which go beyond the examination of overall variance or rhythmic changes in heart rate [2,3]. The goal is to quantify temporal complexity by examining the chaoticity of time series in a reconstructed state space. These methods can be applied to fetal heart period time series to examine whether irregular but typical nonlinear dynamical heart rate patterns can be identified. These patterns might then be linked to fetal well-being.

Fetal heart rate monitoring is generally performed with the cardiotocogram (CTG) or the fetal electrocardiogram (FECG). Both methods have certain drawbacks. The former delivers solely the momentary heart rate with a limited accuracy. The latter produces the electric waveform but, due to artifacts and low signal to noise ratio, can only be applied later in pregnancy. As such these methods are not suited for the acquisition of data earlier during gestation and as such do not allow for an analysis of heart period complexity during the course of pregnancy. On the other hand, it has been shown that magnetocardiography can be used to register fetal cardiac activity as early as the beginning of the second trimester with sufficient precision to identify single QRS complexes and that, in comparison to FECG, it is not influenced by the insulating effect of the vernix caseosa between the 28th and 30th week of gestation $[4,5]$. It is therefore possible to quantify fetal heart period with high accuracy, allowing for the analysis of time series of fetal heart beats starting before the 20th week of gestation.

Goal of this study was to combine these new methods in the registration and analysis of fetal cardiac activity in order to examine changes in fetal heart period complexity in the course of pregnancy. If recognizable patterns can be established for normal gestational development then these methods will open up new diagnostic possibilities which have the added advantage of being completely non invasive.

\section{Methods}

Subjects: We investigated eleven women who presented with healthy singleton pregnancies in the second trimester. Goal was to perform magnetocardiographic registration of fetal cardiac activity (FMCG) at regular intervals of roughly four weeks up to the time of birth. In order to investigate the time course at least three measurements with data amenable to evaluation were required for each fetus. On that basis three fetuses were excluded from the study. One further data set was excluded because gestosis developed. Of the remaining seven fetuses, 36 recordings were acquired between the 16th and 40th week of gestation. The quality of five recordings, all before the 20th week of gestation, was too poor for complexity examination, leaving 31 for evaluation. The mean number of recordings per fetus was $4.4 \pm 1.1$ ( \pm standard deviation, range $3-6$ ), mean time between recordings was $4.1 \pm 2.2$ weeks (range 111). Due to calculation restrictions, the parameter ApD1 could only be calculated for 24 recordings.

Data Acquisition: FMCG recordings were performed with a 37 channel biomagnetometer (Siemens Krenikon). The diameter of the probe is $19 \mathrm{~cm}$. Each sensor consists of a first order gradiometer with a $7 \mathrm{~cm}$ baseline. System noise is less than $30 \mathrm{fT} / \mathrm{Hz}^{-1}$ for frequencies $>2 \mathrm{~Hz}$ and $10 \mathrm{fT} / \mathrm{Hz}^{-1}$ for frequencies $>10 \mathrm{~Hz}$. To reduce the influence of electromagnetic artifacts, the measurements were performed in a shielded room (Vakuumschmelze). The position of the probe was placed over the maternal abdomen such that the fetal signal was optimized. By recording a limb lead maternal ECG the fetal QRS complexes could be clearly identified. Data was recorded for five minutes with a bandpass of $1-200 \mathrm{~Hz}$ at a sampling rate of $1 \mathrm{kHz}$.

Signal analysis: In order to extract the fetal heart period, fetal QRS complexes were marked using an appropriate template in a channel of raw data with strong fetal signal and minimal interference of maternal components. 
Van Leeuwen, et al. MCG and fetal HR complexity. In: Aine C, Okada YC, Stroink G, Swithenby S, Wood C, eds. Advances in Biomagnetism Research: Biomag '96. New York: Springer, 1998: in print

Complexes not automatically recognized were visually identified and added manually; similarly, falsely marked artifacts were removed. Heart period was determined with an accuracy of $\pm 0.5 \mathrm{~ms}$ as the time between consecutive QRS complexes. The time series of a complete set of fetal heart periods served as the basis for further analysis.

Complexity analysis: Two parameters were used to describe the heart dynamics of each fetus at the time of recording: the approximate information dimension (ApD1) and the approximate entropy (ApEn). In contrast to moment statistics, these measures take the order of the data into consideration. Both are based on the correlation integral which reflects the number of data points which are within a defined distance embedded in a $k$-dimensional state space. The complexity measures ApD1 and ApEn characterize the scaling (static) properties of the distribution as well as the expansion behavior (dynamical properties) of the generating dynamics. Methods used in the determination of the approximate dimension and approximate entropy have been described elsewhere [2,6-8]. Apart from the original definitions, we used a strictly local approach for the approximation of the information dimension and the Kolmogorov entropy. By strictly local we mean the computation of local or pointwise dimensions and entropies for reference points generally with their symmetric neighborhood in time. The local approximate dimensions and the local approximate entropies were averaged over the entire data set and provided the values for ApD1 and ApEn (see Appendix for details).

ApD1 and ApEn were determined for each time series. Furthermore, time domain statistics in the form of the mean beat duration, the standard deviation (SD) and the root mean square of successive differences (RMSSD) were also calculated. For the calculation of the time domain statistics, outliers were visually identified in the heart period plot and eliminated from the time series.

In order to examine the dependency of the parameters on gestational age (GA), simple linear regression analysis was performed for each parameter for each fetus over week of gestation. The slope of the regression was taken as a measure of the degree of change of each parameter over time. All values are expressed as mean \pm SD. Linear regression analysis was performed according to the method of least squares. $\mathrm{r}^{2}$ was determined to control the quality of the linear model.

\section{Results}

The number of beats registered per five-minute recording ranged from 629 to 964 (mean: $744 \pm 65$ ). The average beat duration was $412 \pm 24 \mathrm{~ms}$ (heart rate: $146 \pm 9 \mathrm{bpm}$ ). The values of beat period, SD, RMSSD, ApEn and ApD1 tended to increase for most fetuses during pregnancy (see Fig. 1).
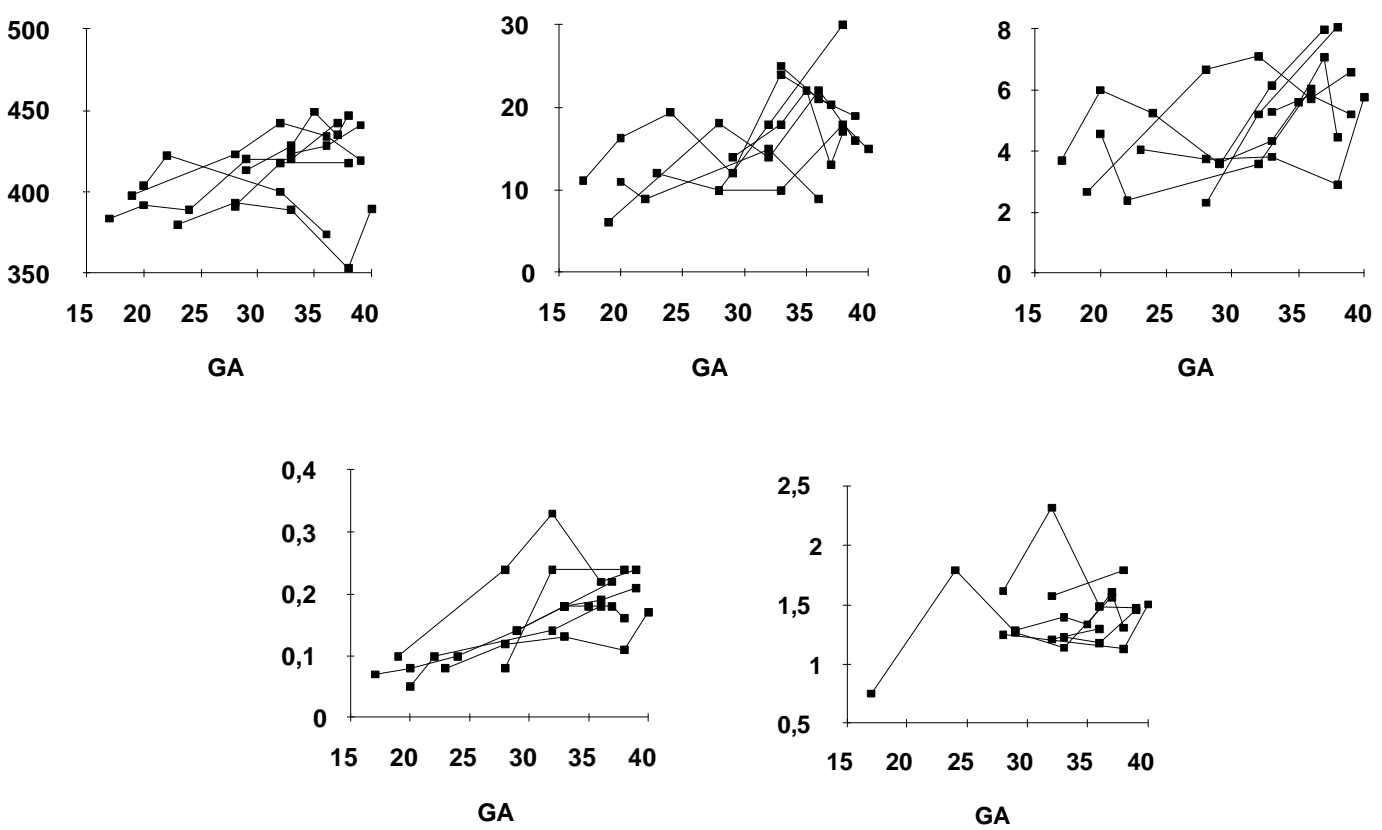

Fig. 1: Course of beat period, SD, RMSSD, ApEn and ApD1 for each fetus with respect to gestational age (GA).

Regression analysis indicated that there was a clear increase in beat period over the gestational period observed for five of the fetuses (Table 1), reflecting a decrease in heart rate of approximately $25 \mathrm{bpm}$. Increases in the time domain measures of HRV, SD and RMSSD, were measurable on the basis of positive slopes in six subjects. 
Van Leeuwen, et al. MCG and fetal HR complexity. In: Aine C, Okada YC, Stroink G, Swithenby S, Wood C, eds. Advances in Biomagnetism Research: Biomag '96. New York: Springer, 1998: in print

However, the $\mathrm{r}^{2}$ values were generally low. In all seven fetuses the slopes for ApEn were positive, for ApD1 in six, indicating an increase in complexity in the course of pregnancy. In particular, the results for ApEn demonstrated high $\mathrm{r}^{2}$ values; the lower correlation results for ApD1 may be due to the lower number of observations.

Table 1: Slope and $\mathrm{r}^{2}$ of the linear regression of beat period, SD, RMSSD, ApD1 and ApEn with respect to gestational age for each subject.

\begin{tabular}{|c|c|c|c|c|c|c|}
\hline ID & $\mathrm{n}$ & beat period $/ \mathrm{r}^{2}$ & $\mathrm{SD} / \mathrm{r}^{2}$ & RMSSD / ${ }^{2}$ & $\mathrm{ApEn} / \mathrm{r}^{2}$ & ApD1 / $r^{2}$ \\
\hline Subj. 1 & 4 & $-2.09 / 0.66$ & $0.07 / 0.03$ & $0.11 / 0.31$ & $0.007 / 0.90$ & $0.03 /$ \\
\hline Subj. 2 & 5 & $-0.64 / 0.08$ & $0.32 / 0.43$ & $0.04 / 0.07$ & $0.004 / 0.58$ & $0.01 / 0.13$ \\
\hline Subj. 3 & 5 & $3.52 / 0.73$ & $0.14 / 0.02$ & 0.24 / 0.39 & $0.003 / 0.32$ & $0.02 / 0.18$ \\
\hline Subj. 4 & 6 & 2.84 / 0.91 & $0.41 / 0.40$ & $0.13 / 0.39$ & $0.008 / 0.97$ & $0.02 / 0.20$ \\
\hline Subj. 5 & 3 & $2.49 / 0.64$ & $2.00 / 1.00$ & $0.57 / 0.98$ & $0.015 / 0.64$ & $0.04 / *$ \\
\hline Subj. 6 & 3 & $2.83 / 0.91$ & $-1.00 / 0.96$ & $-0.01 / 0.02$ & $0.005 / 0.96$ & $0.04 / 0.59$ \\
\hline Subj. 7 & 5 & $1.41 / 0.43$ & $0.58 / 0.58$ & $0.17 / 0.57$ & $0.007 / 0.42$ & $-0.03 / 0.15$ \\
\hline mean $\pm \mathrm{SD}$ & & $1.48 \pm 2.09$ & $0.36 \pm 0.89$ & $0.18 \pm 0.19$ & $0.007 \pm 0.01$ & $0.02 \pm 0.02$ \\
\hline Min / Max & & $-2.09 / 3.52$ & $-1.00 / 2.00$ & $-0.01 / 0.57$ & $0.003 / 0.015$ & $-0.03 / 0.04$ \\
\hline
\end{tabular}

$\mathrm{SD}$, standard deviation; RMSSD, root mean square of successive differences; ApD1, approximate dimension; ApEn, approximate entropy.

*No calculation of $\mathrm{r}^{2}$ because $<3$ values for estimation of regression model.

\section{Discussion}

Analogous to time and frequency domain analysis, complexity analysis can be understood as a time series analysis in the state space domain. The generating dynamics in state space possesses folding and stretching properties which may be analysed by appropriate dynamical measures. ApD1 and ApEn characterize both the scaling (static) properties and the expansion behavior (dynamical properties) of the generating dynamics. Application of these measures on physiological parameters have demonstrated their ability to discriminate between states. For example, the examination of heart rate and blood pressure variability in adults have shown decreasing complexity with age [7].

Several studies have been done on fetuses during labor, applying these analysis techniques on data acquired by scalp ECG. The feasibility of dimensional analysis was thus demonstrated by Chaffin et al [8]. Also, on the basis of ApEn, a reduction in heart rate complexity could be shown in fetuses with acidosis and distress [3]. In contrast, time domain variables such as near-term HRV and mean heart rate did not distinguish between the groups. Furthermore, a decrease in ApEn after drug administration could be documented.

These studies suggest that complexity measures may be a sensitive analytic tool in quantifying fetal heart rate complexity. Their application during pregnancy depends on the possibility of acquiring data with high temporal resolution. At present cardiotocography is the clinical method of choice in the monitoring of fetal heart rate. However it is not suited for analysis of complexity due to limitations in accuracy. The precision in the determination of fetal heart beat duration can in principle be greatly improved by electrocardiography but, among other things, practical problems in signal acquisition and processing have hampered implementation during pregnancy. Our results and other studies, e.g.[4,5], show that FMCG enables the simple and effective acquisition of fetal data. The high quality of the magnetocardiographic signal permits the construction of a time series with the necessary temporal accuracy as early as the second trimester.

The analysis of the data collected here showed that the complexity of fetal heart rate increased throughout the second and third trimester of normal pregnancy. In particular, ApEn was very consistent and its reliability was reflected in high correlation values. ApD1 increased for all but one fetus over time, although the results must be viewed with caution on the basis of the $r^{2}$ values. However, these low correlations may well be due to the lower number of values that could be calculated. This disadvantage will be overcome in future by a different approach in the analysis. As both parameters are independent of the mean and variance of the signal, they have advantages over time domain statistics: they are unaffected by noise, robust to outliers and model independent [6]. In contrast, in order to obtain reasonable values for beat period, SD and RMSSD from the data recorded here, the elimination of outliers was necessary. In spite of this correction of the data, the results for these statistical measures were not as consistent as the complexity measures. Nonetheless, it must be noted that, in the analysis of HRV, such measures are complementary [6] and reflect different aspects of the structure of the time series.

In summary, FMCG may be used as a method to obtain data amenable to the analysis of the temporal complexity of the fetal cardiac system. The observed increase in complexity during the course of pregnancy may be interpreted in terms of the development of the cardiac system. The degree to which this reflects the maturation of the 
Van Leeuwen, et al. MCG and fetal HR complexity. In: Aine C, Okada YC, Stroink G, Swithenby S, Wood C, eds. Advances in Biomagnetism Research: Biomag '96. New York: Springer, 1998: in print

autonomic nervous system and the growth of the fetus as a whole is still to be determined. Further studies with more observations are required to define norm values and to examine the effect of pathological conditions.

\section{References}

[1] Cerutti, S.S., Baselli, G., Civardi, S., Ferrazzi, E., Marconi, A.M., Pagani, M., Pardi, G. Variability analysis of fetal heart rate signals as obtained from abdominal ECG recordings, J Perinat Med, 1986, 14: 445-452.

[2] Kaplan, D.T., Furman, M.I., Pincus, S.M. Techniques for analyzing complexity in heart rate and beat-to-beat blood pressure signals, In: IEEE Comput Cardiol, Los Alamitos, CA, IEEE Computer Society Press, 1990: 243246.

[3] Pincus, S.M. , Viscarello, R.R. Approximate Entropy: A Regularity Measure for Fetal Heart Rate Analysis, Obstet Gynecol, 1992; 79: 249-255.

[4] Dunajski, Z., Peters, M. Development of the magnetocardiograms from the 13th week of gestation onward. In: Baumgartner, C., Deecke, L., Stroink, G., Williamson, S.J. Biomagnetism: Fundamental Research and Clinical Applications, Amsterdam, Elsevier/IOS Press, 1995: 340-341.

[5] Quinn, A., Weir, A., Shahani, U., Bain, R., Maas, P., Donaldson, G. Antenatal fetal magnetocardiography: a new method for fetal surveillance? Br J Obstet Gynaecol, 1994, 101: 866-870.

[6] Pincus, S.M. Quantification of evolution from order to randomness in practical time series analysis, Methods in Enzymology, 1994, 240: 68-89.

[7] Kaplan, D.T., Furman, M.I., Pincus, S.M., Ryan, S.M., Lipsitz, L.A., Goldberger, A.L. Aging and the complexity of cardiovascular dynamics, Biophys J ,1991, 59: 945-9.

[8] Chaffin, D.G., Goldberg, C.C., Reed, L.R. The dimension of chaos in the fetal heart rate, Am J Obstet Gynecol, 1991,165: 1425-1429.

\section{Appendix}

Without loss of generalisation, we interpret the RR-tachogram as a 'time' series with an one beat delay. $k$ successive RR-intervals form a vector in the reconstructed state space indexed by the beat number of the leading RRinterval:

$$
\overrightarrow{R R}_{\mathrm{i}}=\left(\mathrm{RR}_{\mathrm{i}}, \mathrm{RR}_{\mathrm{i}+1}, \ldots, \mathrm{RR}_{\mathrm{i}+\mathrm{k}-1}\right) \in \text { reconstructed state space. }
$$

Both ApEn and ApD1 are based on the correlation integral $\mathrm{C}_{\mathrm{i}}^{\mathrm{k}}(l)$ which is defined as the relative number of $k$-dimensional embedded data points within a $l$-sphere around a reference point with index $i$, as a function of the distance $l$ :

$$
\mathrm{C}_{\mathrm{i}}^{\mathrm{k}}(l)=\frac{1}{\mathrm{~N}-\mathrm{k}+1}\left\{\text { number of vectors } \overrightarrow{\mathrm{RR}}_{\mathrm{j}} \text { with }\left|\overrightarrow{R R}_{\mathrm{i}}-\overrightarrow{\mathrm{RR}}_{\mathrm{j}}\right|<l\right\}, \mathrm{j} \in\left[\mathrm{i}-\operatorname{trunc} \frac{\mathrm{N}}{2}, \mathrm{i}-\mathrm{k}+1+\operatorname{trunc} \frac{\mathrm{N}}{2}\right]
$$

For the determination of the pointwise approximation of fetal heart beat period complexity the following parameter values were set:

- $\quad \mathrm{N}=501 \mathrm{RR}$-intervals. This allowed the calculation of roughly 150-350 local complexity values per recording.

- Embedding dimension: $k=3$.

- Fixed tolerance for ApEn: $l=10 \mathrm{~ms}$.

- The local approximate dimension is defined as the slope of the mean of the logarithmic local correlation integrals between two length scale values $l_{a}$ and $l_{b}$ which are chosen such that

$$
\log _{10} \mathrm{C}_{\mathrm{i}}^{\mathrm{k}}\left(l_{a}\right)=-2.5 \text { and } \log _{10} \mathrm{C}_{\mathrm{i}}^{\mathrm{k}}\left(l_{b}\right)=-0.5 \text {. }
$$

